\title{
Discrete Optimal Multirate Techniques for Excitation Controller Design of a Synchronous Machine
}

\author{
D. I. Pappas" ${ }^{*, 1}$, V. A. Boglou ${ }^{2}$ and S. K. Goumas ${ }^{1}$ \\ ${ }^{1}$ Eastern Macedonia and Thrace Institute of Technology, Ag. Loukas, 65404 Kavala, Greece. \\ ${ }^{2}$ School of Electronics and Computing Engineering, Technical University of Crete, 73100, Chania, Greece.
}

Received 28 January 2016; Accepted 10 February 2016

\begin{abstract}
An optimal control strategy based on Two-Point-Multirate Controllers (TPMRCs), is used to design a desirable excitation controller of a hydrogenerator system, in order to enhance its dynamic stability characteristics. In the TPMRCs based scheme, the control is constrained to a certain piecewise constant signal, while each of the controlled plant outputs is detected many times over a fundamental sampling period T0. On the basis on this strategy, the original problem is reduced to an associate discrete-time linear quadratic (LQ) regulation problem for the performance index with cross product terms, for which a fictitious static state feedback controller is needed to be computed. Simulation results for the actual 117 MVA synchronous generator with conventional exciter supplying line to an infinite grid show the effectiveness of the proposed method which has a quite satisfactory performance.
\end{abstract}

Keywords: Digital Multirate controllers, LQ control, power systems, turbogenerators.

\section{Introduction}

The typical control problem has always been to start with a suitable linear (or linearized) open-loop mathematical model of a physical plant (in continuous or discrete form) and attempt to design a proper controller for it, i.e. to obtain an associated closed-loop system with enhanced dynamic stability characteristics. [1-7]. The digital controller applied for the discrete linear systems may be obtained by using a new TPMRCs $[8,9]$.

It is pointed out that the used TPMRCs technique reduced the original LQ regulation problem to an associated discretetime LQ regulation problem for the performance index with crossed product terms, for which is computer a fictitious static state feedback controller [11-16]. In addition, thus technique offers more flexibility in choosing the sampling rates and provides a power design computed method.

In the present work the discrete linear open-loop system model under consideration systematically derived from the associated continuous 8th order SIMO linearized open-loop model of a practical power system, hawing on 117 MVA synchronous generator supplying power to an infinite grid through a step-up transformer and a transmission line [17, 18]. The sought digital controller for the enhancement of the dynamic characteristics of the above 6 th order discrete model is accomplished by the proper application of the new TPMRCs to it.

\footnotetext{
*E-mail address: dpappas@teiemt.gr ISSN: 1791-2377 @ 2016 Eastern Macedonia and Thrace Institute of Technology. All rights reserved.
}

\section{Overview of Relevant Mathematical Considerations}

Consider the controllable and observable continuous linear state-space system model of the general form

$$
\begin{aligned}
& \mathbf{x}(t)=\mathbf{A x}(\mathrm{t})+\mathbf{B u}(\mathrm{t})(1) \\
& \mathbf{y}(t)=\mathbf{C x}(t)+D \mathbf{u}(t),
\end{aligned}
$$

where: $\mathbf{x}(\mathrm{t}) \in \mathbf{R}^{\mathbf{n}}, \mathbf{u}(\mathrm{t}) \in \mathbf{R}^{\mathbf{m}}$, are the state, input, and controlled output vectors, respectively. In Eqn. 1 all matrices have real elements and appropriate dimensions. Now follows a useful definition.

The associated general discrete description of the system of Eqn. 1 is as follows

$$
\begin{aligned}
& \mathbf{x}(\mathrm{k}+1)=\mathbf{A x}(\mathrm{k})+\mathbf{B u}(\mathrm{k}) \\
& \mathbf{y}(\mathrm{k})=\mathbf{C x}(\mathrm{k})
\end{aligned}
$$

where: $\mathbf{x}(k) \in \mathbf{R}^{\mathbf{n}}, \quad \mathbf{u}(k) \in \mathbf{R}^{\mathbf{m}}, \quad \mathbf{y}(k) \in \mathbf{R}^{\mathbf{p}}$ are state, input and output vectors respectively; and $\mathbf{A}, \mathbf{B}$ and $\mathbf{C}$ are real constant system matrices with proper dimensions.

\section{Overview of new TPMRCs for Linear Discrete Systems}

This method with $\mathrm{H}_{\mathrm{o}}$ and $\mathrm{H}_{\mathrm{N}}$ being zero-order holds and with holding times $T_{o}$ and $T_{N}$, respectively (see Figure 1) is presented here in a concise manner, whereas the details are found [8]. 


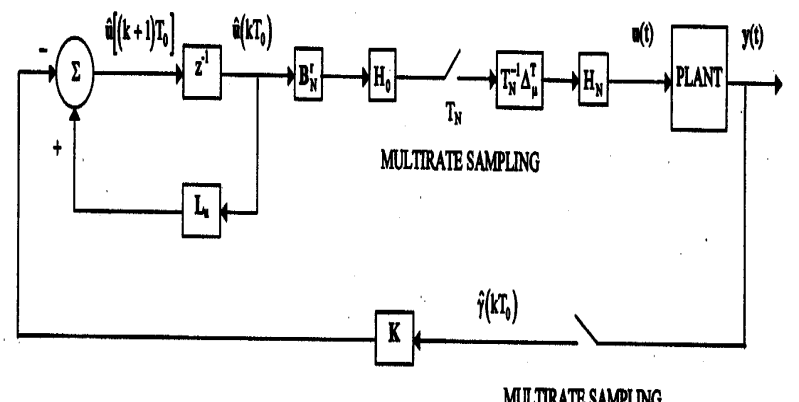

MULTRRATE SAMPLNG

Fig. 1. Simplified representation of power system under investigation in discrete form.

Starting with the general linear state space system description in continuous form

$$
\begin{aligned}
& \mathbf{x}(t)=\mathbf{A} \mathbf{x}(t)+\mathbf{B} \mathbf{u}(t) \\
& \mathbf{y}(t)=\mathbf{C} \mathbf{x}(t)
\end{aligned}
$$

where $\mathbf{x}(\mathrm{t}) \in \mathbf{R}^{\mathbf{n}}, \mathbf{u}(\mathrm{t}) \in \mathbf{R}^{\mathbf{m}}$ and $\mathbf{y}(\mathrm{t}) \in \mathbf{R}^{\mathbf{p}}$ are the state, input and output vectors respectively.

The associated discrete system description is obtained by letting $\mathrm{n}_{\mathrm{i}}, \mathrm{i} \in \mathrm{J}_{\mathrm{p}}=\{1,2, \ldots, \mathrm{p}\}$, be used of observability indices of the pair $(\mathbf{A}, \mathbf{C})$, and $\mathbf{T}_{\mathbf{0}} \in \mathbf{R}^{+}$be a sampling period. Also, by letting

$\Phi=\exp \left(\mathbf{A T}_{0}\right)$

and $\mathbf{B}_{\mathrm{N}} \in \mathbf{R}^{\mathrm{nxp}}$ be the full rank matrix defined by

$$
\mathbf{B}_{\mathrm{N}} \mathbf{B}_{\mathrm{N}}^{\mathrm{T}}=\mathbf{W}_{\mathrm{N}}\left(\mathrm{T}_{0}, 0\right) \geq 0
$$

with the generalized reachability Grammian of ord $\mathrm{N}$ in the interval $\left[0, T_{0}\right]$ being

$$
\begin{aligned}
& \mathbf{W}_{N}\left(T_{0}, 0\right)=T^{*-1} \sum_{\mu=0}^{N_{0}-1} \Delta_{\mu} \Delta_{\mu}^{T}, p_{N}=\operatorname{rank} \mathbf{W}_{N}\left(T_{0}, 0\right) \text { and } \\
& T^{*}=T_{0} / N_{0}, \Delta_{\mu}=\hat{A}_{N}^{N_{0}-\mu-1} \hat{B}_{T^{*}} \\
& \hat{\mathbf{A}}_{N}=\exp \left(\mathbf{A} T^{*}\right), \hat{\mathbf{B}}_{T^{*}}=\int_{0}^{T^{8}} \exp (\mathbf{A} \lambda) \mathbf{B} d \lambda
\end{aligned}
$$

Next follows the application of the OMCM technique to the above descriptions. The input of the plant are constrauned to the following piecewise constant control

$$
\begin{aligned}
& \mathbf{u}\left(k T_{0}+\mu T^{*}+\varsigma\right)=T^{*-1} \Delta_{\mu}^{T} \mathbf{B}_{N}^{\tau} \hat{u}\left(k T_{0}\right), \\
& \hat{\mathbf{u}}\left(k T_{0}\right) \in R^{p_{N}}
\end{aligned}
$$

for

$t=k T_{0}+\mu T^{*}, \mu=0, \ldots, N_{0}-1, k \gg 0$

and $J \in\left[0, T^{*}\right)$, where $B_{N}^{\tau}=B_{N}\left(B_{N}^{T} B_{N}\right)^{-1}$.
The $i^{\text {th }}$ plant output $\mathbf{y}_{\mathbf{i}}(\mathbf{t})$ is detected at every $T_{i}=T_{0} / M_{i}$, such that

$y_{i}\left(k T_{0}+\rho T_{i}\right)=c_{i}^{T} x\left(k T_{0}+\rho T_{i}\right)$,

$\rho=0,1, \ldots, M_{i}-1$

where $M_{i} \in Z^{+}, i \in J_{p}$ are the output multiplicities of the sampling. In general $M_{i} \neq N$. The sampled values of the plant outputs obtained over $\left[k T_{0},(k+1) T_{0}\right)$ are stored in the $M^{*}$-dimentional column vector $\hat{\gamma}\left(k T_{0}\right)$ of the form

$$
\begin{aligned}
\hat{\gamma}\left(k T_{0}\right)= & {\left[y_{1}\left(k T_{0}\right) \ldots y_{1}\left(k T_{0}+\left(M_{1}-1\right) T_{1}\right) \ldots\right.} \\
& \left.y_{p}\left(k T_{0}\right) \ldots y_{p}\left(k T_{0}+\left(M_{p}-1\right) T_{p}\right)\right]^{T}
\end{aligned}
$$

where $M^{*}=\sum_{i=1}^{p} M_{i}$.

The vector $\hat{\gamma}\left(k T_{0}\right)$ is used in the control law of the form

$\hat{\mathbf{u}}\left[(k+1) T_{0}\right]=\mathbf{L}_{u} \hat{\mathbf{u}}\left(k T_{0}\right)-\mathbf{K} \hat{\gamma}\left(k T_{0}\right)$

where $\mathbf{L}_{u} \in \mathbf{R}^{p_{N} x p_{N}}, \quad \mathbf{K} \in \mathbf{R}^{p_{N} x M^{*}}$.

Finally, one scetcs a controller in the form of (6) and (8) which, nowen applied to system (1), minimizes the following performance index

$J=\frac{1}{2} \int_{0}^{\infty}\left[\mathbf{y}^{T}(t) \mathbf{Q y}(t)+\mathbf{u}^{T}(t) \mathbf{R u}(t)\right] d t$

where $\mathbf{Q} \in R^{p x p}$ and $\mathbf{R} \in R^{m x m}$ are symmetric matrices with $\mathbf{Q} \geq 0, \mathbf{R}>0$ while $\left(A \mathbf{C}^{T} \mathbf{Q C}\right)$ is an observable pair.

The above problem is equivalent to the problem of designing a control law of the form of Eqn. 9, in order to minimize the following index:

$$
J=\frac{1}{2} \sum_{k=0}^{\infty}\left[\begin{array}{ll}
\mathbf{x}^{T}\left(k T_{0}\right) & \hat{\mathbf{u}}^{T}\left(k T_{0}\right)
\end{array}\right]\left[\begin{array}{ll}
\mathbf{Q}_{N} & \mathbf{G}_{N} \\
\mathbf{G}_{N}^{T} & \Gamma_{N}
\end{array}\right]\left[\begin{array}{l}
\mathbf{x}\left(k T_{0}\right) \\
\hat{\mathbf{u}}\left(k T_{0}\right)
\end{array}\right]
$$

for the system

$\mathbf{x}\left[(k+1) T_{0}\right]=\Phi \mathbf{x}\left(k T_{0}\right)+B_{N} \hat{u}\left(k T_{0}\right)$

Where $\mathbf{Q}_{N}, \mathbf{G}_{N}, \Gamma_{N}$ are giver explicitly [2].

Theorem 2.1. The following basic formula of the multirate sampling mechanism holds.

$\mathbf{H x}\left[(k+1) T_{0}\right]=\hat{\gamma}\left(k T_{0}\right)-\mathbf{D} \hat{\mathbf{u}}\left(k T_{0}\right), k \geq \mathbf{0}$

where, matrices

$\mathbf{x}\left(k T_{0}+\rho T_{i}\right)=\hat{\mathbf{A}}_{i}^{\rho-M_{i}} \mathbf{x}\left[(k+1) T_{0}\right]+\hat{\mathbf{B}}_{i, \rho} \hat{\mathbf{u}}\left(k T_{0}\right)$

are defined as follows 
$\mathbf{H}=\left[\begin{array}{c}\mathbf{c}_{1}^{T}\left(\hat{\mathbf{A}}_{1}^{M_{1}}\right)^{-1} \\ \mathbf{c}_{1}^{T} \hat{\mathbf{A}}_{1}^{-1} \\ \mathbf{c}_{p}^{T}\left(\hat{\mathbf{A}}_{p}^{M_{p}}\right)^{-1} \\ \mathbf{c}_{p}^{T} \hat{\mathbf{A}}_{p}^{-1}\end{array}\right], \mathbf{D}=\left[\begin{array}{c}\mathbf{c}_{1}^{T} \hat{\mathbf{B}}_{1,0} \\ \mathbf{c}_{1}^{T} \hat{\mathbf{B}}_{1, M_{1}-1} \\ \mathbf{c}_{p}^{T} \hat{\mathbf{B}}_{p, 0} \\ \mathbf{c}_{p}^{T} \hat{\mathbf{B}}_{p, M_{p}-1}\end{array}\right]$

and where,

$y_{i}\left(k T_{0}+\rho T_{i}\right)=\mathbf{c}_{i}^{T} \hat{\mathbf{A}}_{i}^{\rho-M_{i}} \mathbf{x}\left[(k+1) T_{0}\right]+\mathbf{c}_{i}^{T} \hat{\mathbf{B}}_{i, \rho} \hat{\mathbf{u}}\left(k T_{0}\right)$

The ultimate expressions for the control law optimal gain matrices $\mathbf{L}_{u}$ and $\mathrm{K}$ are as follows

$$
\begin{aligned}
& \mathbf{L}_{u}=\left(\mathbf{R}_{N}+\mathbf{B}_{N}^{T} \mathbf{P} \mathbf{B}_{N}\right)^{-1}\left(\mathbf{G}_{N}+\mathbf{B}_{N}^{T} \mathbf{P} \boldsymbol{\Phi}\right) \mathbf{H}^{-1} \mathbf{D} \\
& \mathbf{K}=\left(\mathbf{R}_{N}+\mathbf{B}_{N}^{T} \mathbf{P} \mathbf{B}_{N}\right)^{-1}\left(\mathbf{G}_{N}+\mathbf{B}_{N}^{T} \mathbf{P} \boldsymbol{\Phi}\right) \mathbf{H}^{-1}
\end{aligned}
$$

where $\mathbf{R}_{N}, \mathbf{G}_{N}$ and $\mathbf{H}$ are defined in [2,3]. The resulting discrete closed-loop system matrix $\left(\mathbf{A}_{c l / d}\right)$ takes the following

$$
\mathbf{A}_{c l / d}=\mathbf{A}_{o l / d}-\mathbf{B}_{N} \mathbf{K H}
$$

where $\mathrm{cl}=$ closed-loop, $\mathrm{ol}=$ open-loop and $\mathrm{d}=$ discrete.

\section{Design and Simulations of Resulting Discrete Closed- Loop Power System Model}

In the present work, the aforementioned optimal control strategy is used to design a desirable excitation controller of a hydrogenerator system, for the purpose of enhancing its dynamic stability characteristics. The hydrogenerator system studied, is an 117 MVA hydrogenerator unit of the Greek Electric Utility Power System, and which supplies power through a step-up transformer and a transmission line to an infinite grid. A linear model of the hydrogenerator can be obtained by linearizing its nonlinear Park's equations [16,17] about the operating point, of the hydrogenerator unit are ginen in table 1 wherein $\mathrm{P}_{t}$ and $\mathrm{Q}_{\mathrm{t}}$ denote the active and the reactive generator power.

Table 1. Operating point of the hydrogenerator system.

\begin{tabular}{c|c|c}
\hline $\mathbf{v}_{\mathbf{t}}$ (p.u.) & $\mathbf{P}_{\mathbf{t}}$ (p.u.) & $\mathbf{Q}_{\mathbf{t}}$ (p.u.) \\
\hline 1.0 & 0.4 & -0.68 \\
\hline
\end{tabular}

Based on the state variables Figure 2 and the values of the parameters and the operating point (see Appendix A), the system of Figure 2 may be described in state-space form, in the form of Eqn. 1, where

$$
\begin{aligned}
& \mathbf{x}=\left[\begin{array}{llllll}
\delta & \omega & v_{t} & P_{t} & i_{f} & E_{f d}
\end{array}\right]^{T} \\
& u=\Delta V_{\text {ref. }}, \quad \mathbf{y}=\mathbf{x} .
\end{aligned}
$$

The matrices A, B, C, are given in Appendix B.

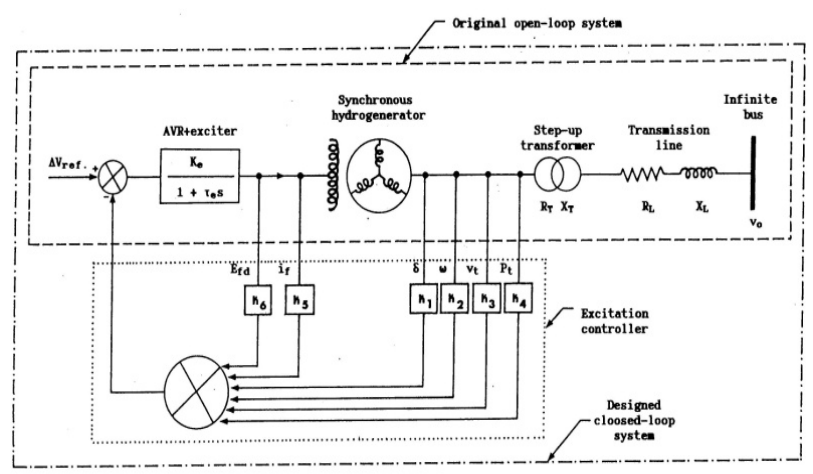

Fig. 2. Simplified representation of investigated practical power system.

The eigenvalues of the original continuous open-loop power system models and the simulated responses of the output variables $\left(\delta, \omega, v_{t}, P_{t}, i_{f}, E_{f d}\right)$, are shown in Table 2 and Figure 3, respectively.

Table 2. Eigenvalues of original open-loop power system

\begin{tabular}{l|rrr}
\multicolumn{4}{c}{ model. } \\
\hline$\lambda$ & -25.7163 & $-1.5764 \pm 9.0779 \mathrm{i}$ & $-7.7698 \pm 5.9411 \mathrm{i}$ \\
& \multicolumn{3}{c}{-3.6592} \\
\hline
\end{tabular}

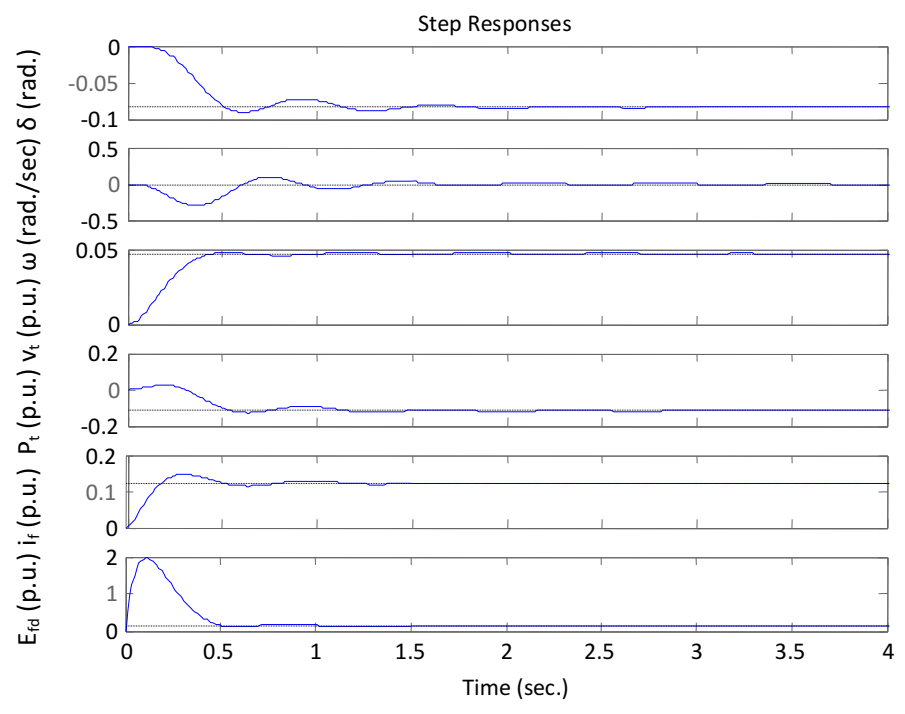

Fig. 3. Responses of the output variables of the original continuous openloop power system models to step input change: $\Delta \mathrm{V}_{\text {ref }}=0.05$.

The computed discrete linear open-loop power system model, based on the associated line arized continuous openloop system model described in Appendix B, is given below in terms of its matrices with sampling period $\mathrm{To}=0.2 \mathrm{sec}$.

$$
\begin{aligned}
A_{\text {ol/d }} & =\left[\begin{array}{cccccc}
-3.9360 & 0.1051 & -3.7891 & 1.8211 & -0.0840 & -0.0029 \\
-23.6256 & -0.1821 & -16.6745 & 8.1062 & -0.7112 & -0.0287 \\
0.3975 & -0.0019 & 0.5291 & -0.1769 & 0.0688 & 0.0032 \\
-8.2682 & 0.2332 & -7.7538 & 3.8983 & -0.0671 & -0.0009 \\
-0.7243 & 0.0611 & -0.8290 & 0.3535 & 0.1208 & 0.0078 \\
-16.0254 & 0.1410 & -29.7656 & 7.3922 & -3.7738 & -0.1391
\end{array}\right] \\
B_{\text {ol/d }} & =\left[\begin{array}{llllll}
-0.1728 & -2.8785 & 0.5197 & 0.4938 & 2.5522 & 30.9687
\end{array}\right]^{T}
\end{aligned}
$$




$$
C_{o l / d}=C=\left[\begin{array}{llllll}
1 & 0 & 0 & 0 & 0 & 0 \\
0 & 1 & 0 & 0 & 0 & 0 \\
0 & 0 & 1 & 0 & 0 & 0 \\
0 & 0 & 0 & 1 & 0 & 0 \\
0 & 0 & 0 & 0 & 1 & 0 \\
0 & 0 & 0 & 0 & 0 & 1
\end{array}\right]
$$

The computed magnitude of the eingenvalues of the discrete open-loop power system models and the simulated responses of the output variables $\left(\delta, \omega, \mathrm{v}_{\mathrm{t}}, \mathrm{P}_{\mathrm{t}}, \mathrm{i}_{\mathrm{f}}, \mathrm{E}_{\mathrm{fd}}\right)$, are shown in Table 3 and Figure 3, respectively.

Table 3:.Magnitude of eigenvalues of discrete original openloop and designed closed-loop power system models.

\begin{tabular}{c|c|ccc}
\hline Original open-loop & $|\lambda|$ & 0.7296 & 0.7296 & 0.4810 \\
power system model & $\mid$ & 0.2114 & 0.2114 & 0.0058 \\
\hline Designed closed- & $|\hat{\lambda}|$ & 0.5359 & 0.5359 & 0.3293 \\
loop power system & 0.1787 & 0.1787 & 0.0057 \\
model & & & & \\
\hline
\end{tabular}

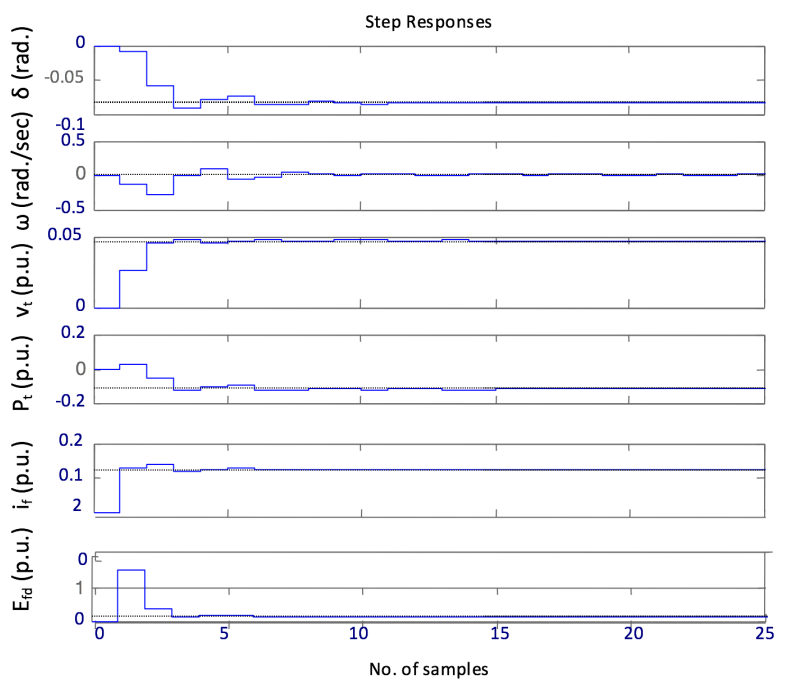

Fig. 4. Responses of the output variables of the original discrete openloop power system model to step input change: $\Delta \mathrm{V}_{\text {ref. }}=0.05$.

Due to space limitations the numerical description of the resulting discrete close loop system model is not presented here, but it depends on the following derive weight matrices $\mathbf{Q}=$ diag.(1 $0.001 \quad 0.01 \quad 0.001 \quad 0.0001 \quad 0.00001)$ and $\mathbf{R}=1$, and the chosen output multiplicities of the sampling:

$\mathrm{M}=\left[\begin{array}{llllll}2 & 4 & 6 & 8 & 10 & 12\end{array}\right], \mathrm{N}=8$.

Evaluation relations (11) and (12) we obtain the admissible TPMRC gains.

The computed values of $\mathbf{B}_{\mathbf{N}}, \mathbf{K}, \mathbf{L}_{\mathbf{u}}$ and feedback gain matrices are:

$$
B_{\mathrm{N}}=\left[\begin{array}{cccccc}
0.5480 & 0 & 0 & 0 & 0 & 0 \\
7.5204 & 2.1725 & 0 & 0 & 0 & 0 \\
-1.0351 & -0.6509 & 0.2221 & 0 & 0 & 0 \\
-0.5465 & -1.0853 & 0.3661 & 0.0007 & 0 & 0 \\
-3.6567 & -3.9961 & 2.3564 & 0.2923 & 0.0840 & 0 \\
24.8707 & -37.7503 & 102.9724 & 69.3662 & 65.3728 & 34.4845
\end{array}\right]
$$

$$
\begin{aligned}
F & =\left[\begin{array}{cccccc}
-0.9806 & 0.0102 & -0.8505 & 0.4129 & -0.0284 & -0.0012 \\
-0.1004 & -0.0084 & -0.0495 & 0.0358 & -0.0094 & -0.0005 \\
-0.2241 & 0.0157 & -0.2399 & 0.0971 & 0.0036 & 0.0004 \\
-0.1127 & 0.0086 & -0.1233 & 0.0492 & 0.0023 & 0.0002 \\
0.0130 & -0.0010 & 0.0142 & -0.0057 & -0.0003 & 0.0 \\
-0.0857 & 0.0047 & -0.0873 & 0.0367 & 0.0005 & 0.0001
\end{array}\right] \\
L_{u} & =\left[\begin{array}{cccccc}
0.1697 & -0.5419 & 0.6411 & -41.5272 & -35.1617 & 0.0215 \\
-0.0539 & 0.0661 & -0.1294 & 18.5269 & 156.4308 & 0.0232 \\
0.1304 & -0.2641 & 0.3599 & -37.3146 & -315.3241 & -0.0271 \\
0.0707 & -0.1412 & 0.1945 & -20.3661 & -172.1022 & -0.0151 \\
-0.0081 & 0.0163 & -0.0225 & 2.3584 & 19.9287 & 0.0018 \\
0.0410 & -0.0871 & 0.1161 & -11.5424 & -97.5518 & -0.0074
\end{array}\right]
\end{aligned}
$$

The magnitude of the eigenvalues of the discrete original open-loop and of the designed closed-loop power system model are shown in Table 3.

The simulated responses of the output variables $\left(\delta, \omega, \mathrm{v}_{\mathrm{t}}\right.$, $\left.\mathrm{P}_{\mathrm{t}}, \mathrm{i}_{\mathrm{f}}, \mathrm{E}_{\mathrm{fd}}\right)$ of the discrete original open-loop and designed closed-loop power system models, for zero initial conditions and unit step input disturbance, are shown in Figure 3 and Figure 4.

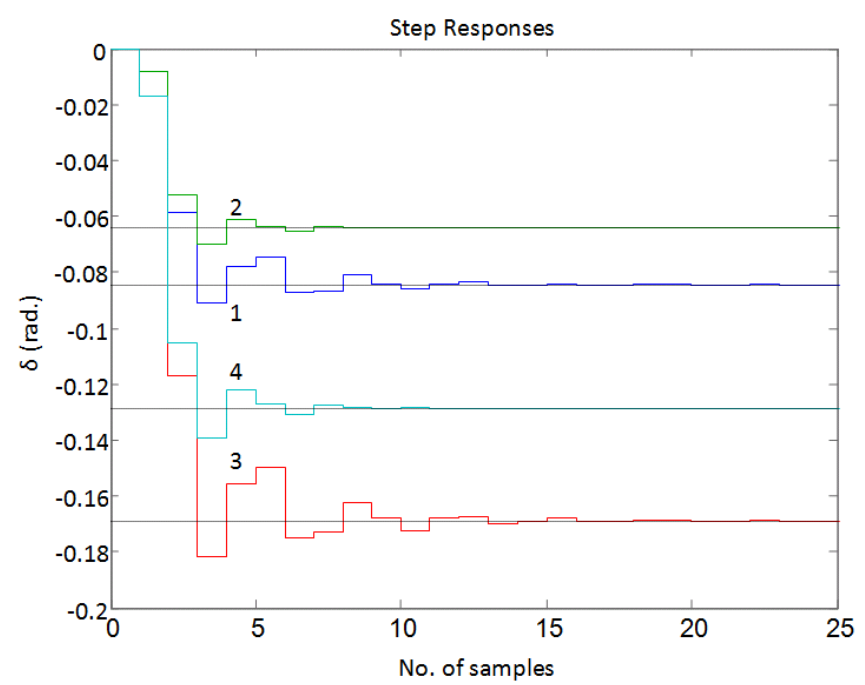

(A)

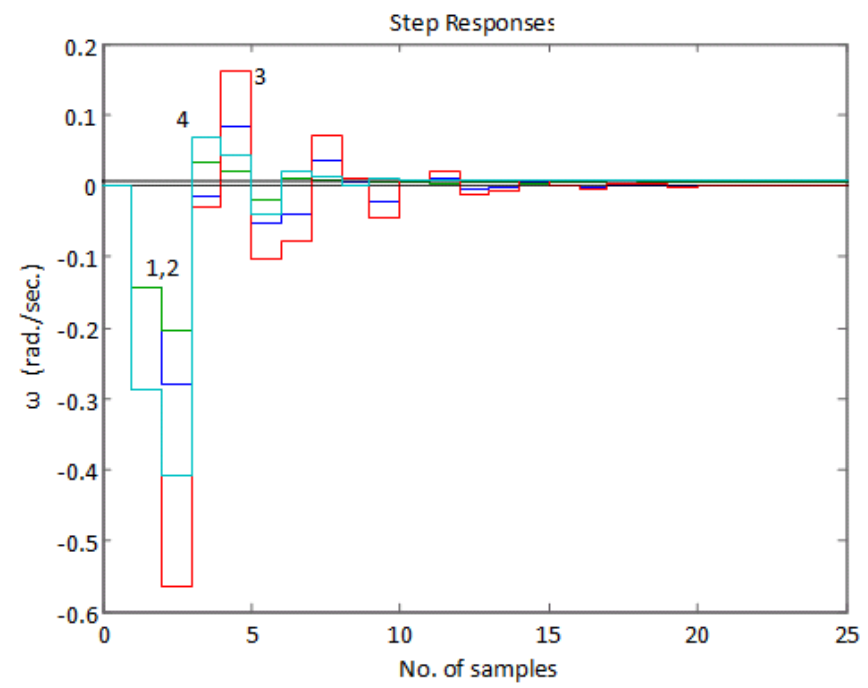

(B) 

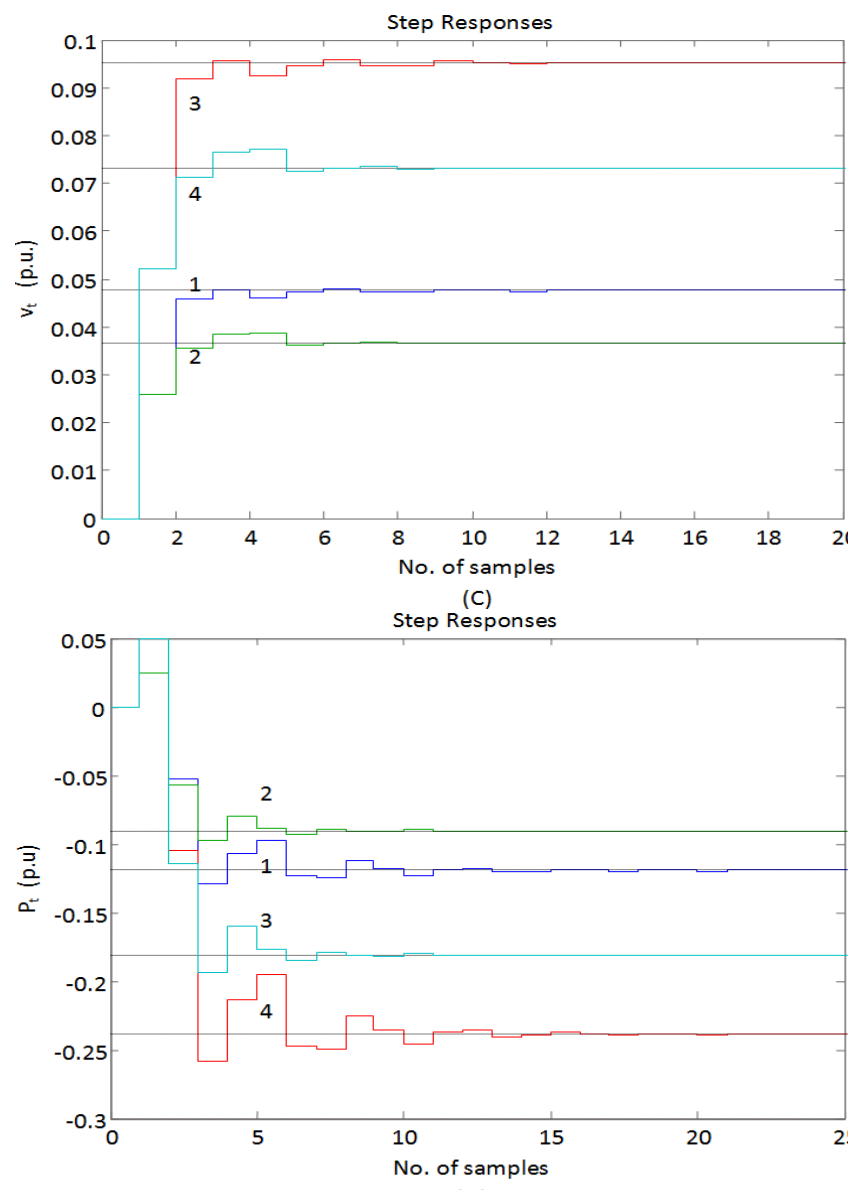

(D)
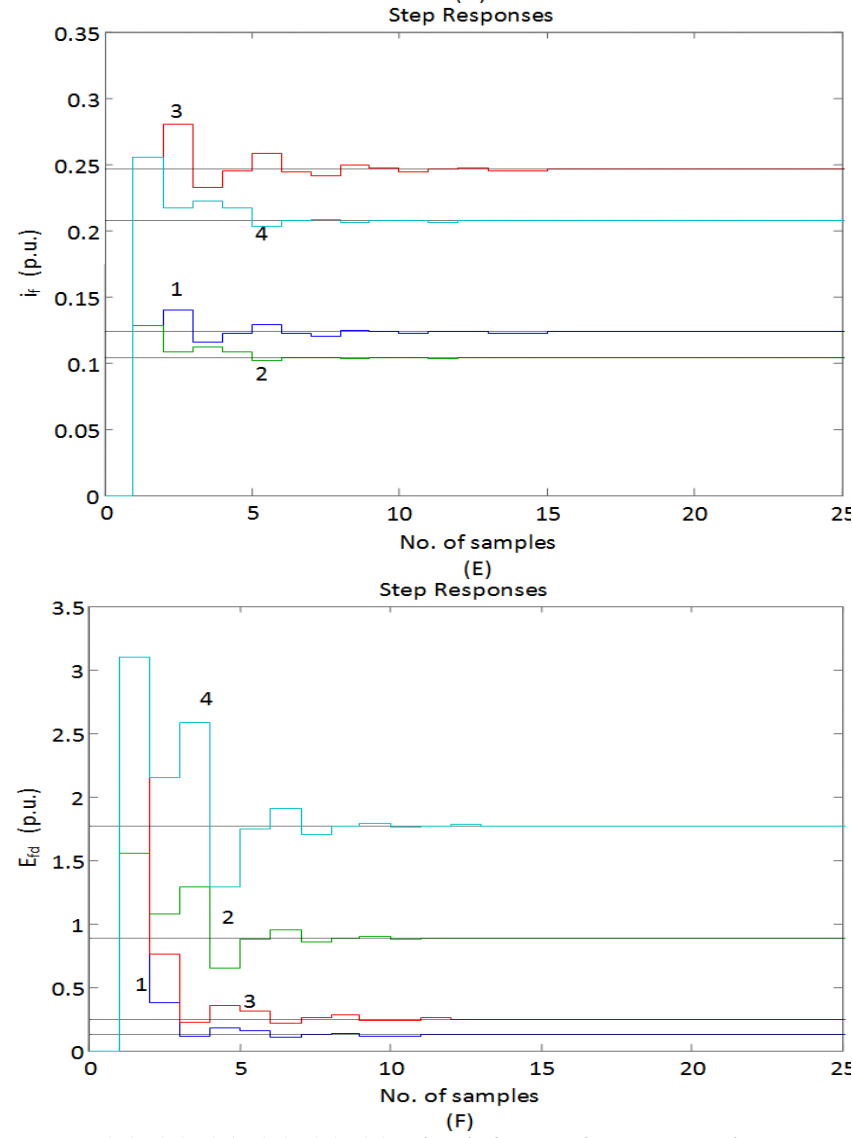

Fig. 5. (A), (B), (C), (D), (E), (F): Simulation results represent the responces of the discrete control system:

(1), (3) for the open loop to step input power load changes $\Delta \mathrm{V}_{\text {ref. }}=0.05$ p.u. and 0.10 p.u. respectively.

(2), (4) for the closed loop to step input power load changes $\Delta \mathrm{V}_{\text {ref. }}=$ 0.05 p.u. and 0.10 p.u. respectively.
By comparing the computed eigenvalues of the simulated responses of the discrete original open-loop power system model and the associated designed discrete closed-loop models, it is clear that the resulting enhancement in the dynamic system stability of the closed-loop system model is remarkable.

From Figure 3 it is clear that the dynamic stability characteristics of the designed discrete closed- loops systemsmodels are far more syperior than the correspondig, ones of the original open-loop model, which attests in favour of the proposed TPMRCs-control technique.

It is to be noted that the solution results of the discrete system models (i.e. eigenvalues, eigenvectors, responses of system variables etc.) for zero initial conditions were obtained using a special software program (which is based on the theory of $\S 3$ and runs on MATLAB program environment).

In Figure 6 , the variation of the optimal average cost $J_{\text {opt }}$ [1] with respect to the fundamental sampling period $T_{0}$ is depicted in the case where (No, M1, M2, M3, M4, M5, $M 6)=(8,2,4,6,7,8,12)$ is depicted. The optimal average cost obtained is given by [2], $J_{\text {opt }}=\frac{1}{2} \mathbf{x}(0)^{T} \mathbf{P x}(0)$, where $\mathbf{P}$ is the Riccati solution [1, 2, 9]. In our case simple calulations yield $J_{\text {opt }}=92.4198$.

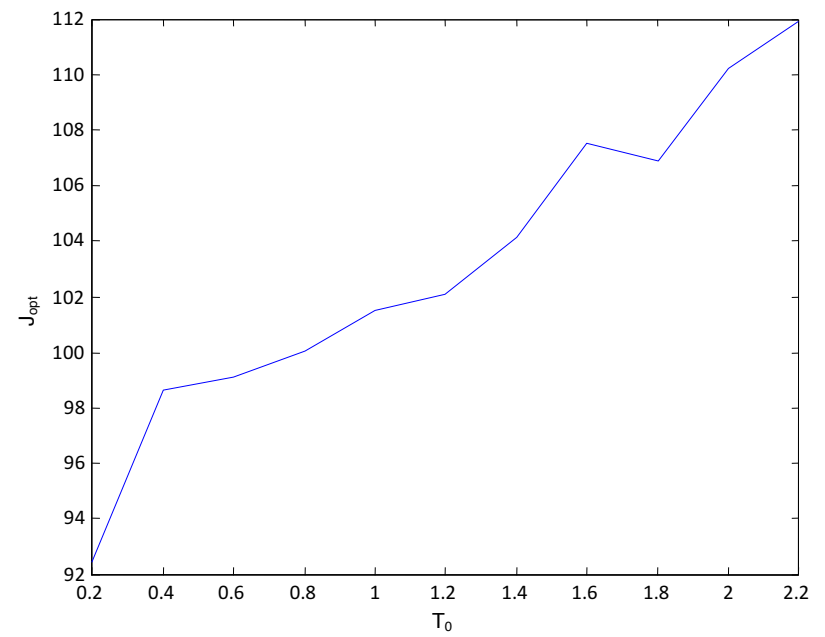

Fig. 6. Discrete-time optimal average cost $\mathrm{J}_{\mathrm{opt}}$ versus sampling $\mathrm{T}_{\mathrm{o}}$

It is to be noted that the solution results of the discrete system models (i.e. eigenvalues eigenvectors, responses of system variables etc) for zero initial conditions were obtained using a special software program (which is based on the theory of $\S 3$ and runs on MATLAB program environment).

\section{Conclusions}

An optimal digital control strategy based on Two-PointMultirate Controllers has been used in this paper in order to design a desirable excitation controller of an unstable hydrogenerator system, for the purpose of enhancing its dynamic stability characteristics. The proposed method offers acceptable closed loop response as well as more design flexibility (particularly in cases where the system states are not measurable), and its performance is at least comparable to known LQ optimal regulation methods. 


\section{References}

1. Hagiwara T., Araki M.: "Design of a stable state feedback controller based on the multirate sampling of the plant output". IEEE Trans. Autom. Control, vol. AC-33, 1988, pp. 812-819.

2. Al-Rahmani H.M. and Franklin G.F., Multirate Control: A new approach, Automatica 28, 1992, pp. 35-44.

3. Arvanitis K. G. "An indirect model reference adaptive controller based on the multirate sampling of the plant output”. Int. J. Adapt. Control Sign. Proc., vol. 10, 1996, pp. 673-705 .

4. Arvanitis K.G. Paraskevopoulos P.N. and Vernardos A.A., "Multirate Adaptive Temperature Control of Greenhouses", Computers and Electronics in Agriculture, vol. 26, 2000, pp. 303320.

5. S. Longhi, "Structural properties of multirate sampled-data systems", IEEE Trans. On Automat. Control, 39 (3), 1994, pp. $692-$ 696.

6. Ogata, K., 1994, "Discrete-time control systems", Prentice Hall, Englewood Cliffs, New Jersey.

7. Boglou A.K and Papadopoulos D.P., "Dynamic performance improvement og hydro generator with modern pole- assignment control methods", Journal Electrical Engineering 46, No 3, 1995, pp. 81-89.

8. Sagfors, M.F., Toivonen, H. T. and Lennartson B., "H ${ }^{\infty}$ Control of Multirate Sampled-Data Systems: A state-space Approach", Automatica, Vol. 34, No. 4, 1998, pp. 415-428.

9. Fujimoto H., Hori Y., "High-performance servo systems based on multirate sampling control", Control Engineering Practice, Vol. 10, Issue 7, 2002, pp. 773-781.
10. Srinivasarao, S. C. Patwardhan, R.D. Gudi, "Nonlinear predictive control of irregularly sampled multirate systems observers", Journal of Process Control, Vol. 17, Issue 1, 2007, pp. 17-35.

11. Qing Wei Jia, "A new method of multirate state feedback control with application to an HDD servo system”, Mechatronics, vol. 18, Issue 1, 2008, pp. 13-20.

12. Mizumoto, T. Chen, Ohdaira S., Zenta M.K., "Adaptive output feedback control of general MIMO systems using multirate sampling and its application to acart-crane system", Automatica, vol. 43, Issue 12, 2007, pp. 2077-2085.

13. Chak C. K., Feng G., Hesketh T., "Multirate adaptive optimal control with application to DC motor", Computers \& Electrical Engineering, vol. 23, Issue 2, 1997, pp. 65-79,.

14. Heidarinejad M., Liu J., D. Munoz de la Pena, Di J.F., "Multirate Lyapunov-based distributed model predictive control of nonlinear uncertain systems", Journal of Process Control, vol. 21, Issue 9, 2011, pp. 1231-1242.

15. Polushin L. G., Marquez H. J., "Multirate of sampled-data stabilization of nonlinear systems", Automatica, vol. 40, Issue 6, 2004, pp. 1035-1041.

16. Cimino M., Pagilla P. R., "Design of linear time-invariant controllers for multirate systems", Automatica, vol. 46, Issue 8, 2010 , pp. 1315-1319.

17. R. H. Park, "Two reaction theory of synchronous machines: General method of analysis”. Part I. Trans AIEE, vol. 48, 1929, pp. 716-727. 


\section{APPENDIX A}

Numerical values of the system parameters and the operating point (p.u. values on generator ratings).

Hydrogenerator:

117MVA, $\mathrm{kV}=15.75, \mathrm{RPM}=125, \mathrm{H}=3.0, \mathrm{x}_{\mathrm{d}}=0.935$ p.u., $\mathrm{x}_{\mathrm{q}}=0.574$ p.u., $\mathrm{x}_{\mathrm{D}}=0.992$ p.u., $\mathrm{x}_{\mathrm{Q}}=0.551$ p.u., $\mathrm{x}_{\mathrm{f}}=0.221$ p.u., $\mathrm{i}_{\mathrm{q}}=0.665$ p.u., $\mathrm{i}_{\mathrm{d}}=0.746$ p.u., $\mathrm{v}_{\mathrm{q}}=0.924$ p.u., $\mathrm{v}_{\mathrm{d}}=0.381$ p.u.

\section{APPENDIX B}

Numerical values of matrices $\mathbf{A}, \mathbf{B}, \mathbf{C}, \mathbf{D}$ of the original continuous $6^{\text {th }}$-order system and $\mathbf{X}(0)$, the vector of the condition initial.

$$
A=\left[\begin{array}{cccccc}
0 & 1 & 0 & 0 & 0 & 0 \\
-520.7453 & 0 & -419.7003 & 201.2473 & 0 & 0 \\
-1.0160 & -0.0463 & -7.8918 & -0.0836 & 2.2300 & 0.0419 \\
16.9059 & 1.9757 & 0.1124 & -8.0416 & 3.6896 & 0.0693 \\
42.0882 & 0.2297 & 66.1062 & -15.2879 & -12.1346 & 0.6412 \\
0 & 0 & -1000 & 0 & 0 & -20
\end{array}\right]
$$$$
\mathbf{X}(0)=\left[\begin{array}{llllll}
0.4914 & 314 & 1 & 0.4 & 1.0107 & 0.4734
\end{array}\right]^{T}
$$

\section{APPENDIX C}

Numerical values of matrices $\mathbf{A}_{\mathrm{cl} / \mathrm{d}}$ and $\mathbf{B}_{\mathrm{cl} / \mathrm{d}}$ closed-loop discrete $6^{\text {th }}$-order system and $\mathbf{P}$ the Riccati solution

$$
\begin{aligned}
\mathbf{A}_{c l / d} & =\left[\begin{array}{cccccc}
-3.3986 & 0.0995 & -3.3230 & 1.5948 & -0.0684 & -0.0022 \\
-16.0331 & -0.2404 & -10.1709 & 4.9231 & -0.4770 & -0.0189 \\
-0.6330 & -0.0003 & -0.3302 & 0.2522 & 0.0324 & 0.0015 \\
-8.8309 & 0.2239 & -8.1844 & 4.1272 & -0.0942 & -0.0023 \\
-4.1511 & 0.0253 & -3.5368 & 1.7636 & -0.0299 & 0.0004 \\
37.5771 & -2.7414 & 24.8519 & -15.8357 & -3.9555 & -0.1899
\end{array}\right] \\
B_{c l / d} & =\left[\begin{array}{ccccccc}
0.5480 & 0 & 0 & 0 & 0 & \\
7.5204 & 2.1725 & 0 & 0 & 0 & 0 \\
-1.0351 & -0.6509 & 0.2221 & 0 & 0 & 0 \\
-0.5465 & -1.0853 & 0.3661 & 0.0007 & 0 & 0 \\
-3.6567 & -3.9961 & 2.3564 & 0.2923 & 0.0840 & 0 \\
24.8707 & -37.7503 & 102.9724 & 69.3662 & 65.3728 & 34.4845
\end{array}\right]
\end{aligned}
$$

External system:

$\mathrm{R}_{\mathrm{e}}=0.015$ p.u., $\mathrm{X}_{\mathrm{e}}=0.40$ p.u. (on a $117 \mathrm{MVA}$ base).

Operating point:

$\mathrm{v}_{\mathrm{to}}=1.0$ p.u., $\mathrm{P}_{\mathrm{t} 0}=1.1$ p.u., $\mathrm{Q}_{\mathrm{t} 0}=0.5$ p.u.,

$\delta_{\text {nom }}=0.9604 \mathrm{rad} ., \omega_{\text {nom }}=100 \pi \mathrm{rad} . / \mathrm{sec}, \mathrm{i}_{\text {fnom. }}=1.9634$ p.u., $\mathrm{E}_{\text {fdnom. }}=1.7720$ p.u.

$$
B=\left[\begin{array}{llllll}
0 & 0 & 0 & 0 & 0 & 1000
\end{array}\right]^{T}
$$$$
C=\left[\begin{array}{llllll}
1 & 0 & 0 & 0 & 0 & 0 \\
0 & 1 & 0 & 0 & 0 & 0 \\
0 & 0 & 1 & 0 & 0 & 0 \\
0 & 0 & 0 & 1 & 0 & 0 \\
0 & 0 & 0 & 0 & 1 & 0 \\
0 & 0 & 0 & 0 & 0 & 0
\end{array}\right]
$$

$$
\mathbf{C}_{c l / d}=\mathbf{C}
$$

$P=\left[\begin{array}{cccccc}4.3830 & -0.0744 & 3.9291 & -1.8932 & 0.1097 & 0.0041 \\ -0.0744 & 0.0024 & -0.0761 & 0.0364 & -0.0015 & 0 \\ 3.9291 & -0.0761 & 3.6309 & -1.7476 & 0.0980 & 0.0036 \\ -1.8932 & 0.0364 & -1.7476 & 0.8419 & -0.0473 & -0.0017 \\ 0.1097 & -0.0015 & 0.0980 & -0.0473 & 0.0030 & 0.0001 \\ 0.0041 & 0 & 0.0036 & -0.0017 & 0.0001 & 0\end{array}\right]$ 